DOI:10.2478/rrlm-2018-0037

\title{
Platelets and Infections
}

\author{
Daniel Emil Albu ${ }^{1}$, Monica Copotoiu ${ }^{2 *}$, Peter Szmuk ${ }^{3}$, Sanda - Maria Copotoiu ${ }^{4}$ \\ 1. Regina Maria, Medical Center, Târgu-Mureş, Romania \\ 2. Department of Rheumatology and Physiotherapy, County Emergency Clinical Hospital, \\ Târgu-Mureș, România \\ 3. University of Texas, South Western, USA \\ 4. Department of ICU and Anesthesiology I, County Emergency Clinical Hospital, \\ Târgu-Mureș, România
}

Received: $16^{\text {th }}$ September 2018; Accepted: $8^{\text {th }}$ October 2018; Published: $9^{\text {th }}$ October 2018

\section{Background}

Our society is characterized by an increasing trend of sepsis - a life-threatening organ dysfunction caused by a dysregulated host response to an infection. [1] According to literature, a total number of 53.9 million adult infection admissions was registered between 2003 and 2009 in the United States of America with an increase of infection claim at admission of $11 \%$ and of sepsis claim at admission of $49 \%$. [2] This trend might be due to antibiotic resistance. New procedures have been implemented in order to lower the hospitalization length, costs and affiliated morbidity and mortality. Certain procedures are under investigation concerning their role in reducing infections. In oral, plastic, cardiac and orthopedic surgery, platelet-rich plasma (PRP) is a local option. New studies have proven not only its regenerative role (first described in early 90's as combination of PRP with fibrin +/- thrombin improving wound healing) but also an antimicrobial one due to the presence of platelets. $[3,4]$

\section{The role of platelets in infection}

Platelets or thrombocytes - anucleate blood elements with a life span of no more than 10 days are known mainly for their role in hemostasis, but they also modulate the immune response. Platelets are described as vascular sentinels due to their high number in circulation as well as expressed glycoprotein receptors. [5]

\section{Players and mechanism of action}

Platelets intervene in inflammatory, immune responses by expressing on their surface protein receptors such as Toll like receptors (TLR), lectins, integrins, Fc receptors directly coming in contact with the antigens (bacteria, viruses etc.) and recognizing immune complexes. [6] They release inflammatory mediators inducing leucocyte proliferation and activation and direct contact with endothelial cells playing an important role in vascular damage resolution and tissue repair.

\footnotetext{
*Corresponding author: Monica Copotoiu, University of Medicine, Pharmacy, Sciences and Thechnology of Târgu Mureş, Department of Rheumatology, Târgu Mureş, Romania .E-mail: monicacopotoiu@gmail.com
} 
Inflammation mediates several processes such as thrombosis and infection. They produce phospholipid vesicles known as platelet microparticles (PMP). PMPs express the same antigens as "the mother platelets" thus being differentiated from other cells (leucocytes, red blood cells, monocytes) derived microparticles. Platelets are to be found in large number in circulation so the PMP represents up to $90 \%$ of the circulating phospholipid vesicles. PMPs are released during apoptosis or senescence of platelets. They are produced also by bacterial infection and express on their surface a pro-coagulant phospholipid - phosphatidylserine (PS), but also the major tissue factor for promoting coagulation. PMPs are involved in immune responses by modulating the activity of chemokines such as RANTES (regulated on activation, normal $\mathrm{T}$ cell expressed and secreted), cytokines - interleukin (IL) - 1ß and proteins such as CD40ligand (CD40L) being involved in co-stimulation of T cell. PMPs can act outside of platelets. [5,7]

The mechanisms of actions between platelets and bacteria are: direct binding to the surface receptors, binding of toxins - bacterial products to platelets and indirect binding of bacteria via a ligand of a platelet receptor - a plasma protein. [5]

The surface receptors involved in the interaction between the bacteria and the platelets are: glycoprotein IIb-IIIa, glycoprotein Ib $\alpha$ and Fc $\gamma$ RIIa receptors. [5]

Glycoprotein IIb-IIIa is involved in the process of adhesion and aggregation of the platelets being the receptor for fibrinogen. Staphylococci express specific fibrinogen like surface receptors. Those proteins can recognize adhesive matrix molecules family (MSCRAMM), thus Staphylococci promote infection by adhering to tissues. The Staphylococci (S) receptors such as $\mathrm{Fbl}$ enabling bacteria to bind with fibrinogen can share common features as MSCRAMM. MSCRAMM have different sites of binding with fibrinogen. [5]
Other bacteria using the same pathway are: Streptococcus pyogenes and Streptococcus mitis.

$S$. epidermidis can by-pass the fibrinogen pathway and binds directly with the Glycoprotein IIb-IIIa receptor. [5]

Glycoprotein Iba (GPIba) is a receptor found only on the surface of platelets and has a high affinity for von Willebrand factor (vWF). Several species of Streptococcus, S. aureus and Helicobacter pylori are able to bind with vWF thus interacting with GPIb $\alpha$.

Fc $\gamma$ RIIa receptor is also found on the surface of neutrophils and monocytes. It recognizes the Fc domain of immunoglobulin (Ig) $G$ enabling the binding and internalization of immune complexes. It is the only type of Fc $\gamma$ receptor described on thrombocytes. His stimulation is required by other platelet receptors in order to get a response to the aggression of bacteria. Due to their number, the platelets are considered to be the number one reservoir for the Fc $\gamma$ RIIa receptors. [5,8]

Complement system can interact with the activated platelets promoting their clearance and as well their activation. Complement interacts with bacteria involving two pathways: the conventional and the alternative pathway. $S$. aureus induces platelet aggregation dependent on complement. Due to complement factors expressed on the surface of the platelets, they can intervene in the destruction of the bacteria by increasing the activity of the complement. On the other hand, due to their complement receptors, platelets can be targets for complement's lytic activity.

Platelets can release antibacterial molecules known as platelet microbicidial proteins (PmP), or thrombocidins. They are not defensins being characterized by different sequence, molecular mass and cationic charges. Bacteria and thrombin are responsible for PmP1 and PmP2 release, thrombin modulating their function as well. Part of PmP family are kinocidins, platelet's cyto- 
kines. Two subgroups of kinocidins have been described: $\alpha$-kinocidins and $\beta$-kinocidins. The $\alpha$-kinocidins are represented by connective tissue activating peptide (CTAP3), neutrophil activating peptide (NAP2), platelet basic protein (PBP) and PF4 known as CXC-cytokines. ß-kinocidins is represented by RANTES, a CC-type cytokine. They exhibit synergetic effect. The presence of PF4 augmented the connective tissue-activating peptide III (CTAP3) on E. coli, but not acting alone. [5,9]

\section{Platelet products used for curative purposes}

The hypothesis of the antibacterial properties of the platelets encouraged the use of autologous plasma concentrates such as platelet-rich plasma (PRP) in preventing wound infections in cardiac, oral, plastic, abdominal and orthopedic surgery.

Concentrated platelet products are referred to in terms such as: platelet rich plasma gel, fibrin clot, autologous fibrin glue, platelet rich plasma, platelet and leucocyte rich plasma (L-PRP) etc. There are different classifications of PRPs according to preparation protocols. The classification of plasma concentrates that is accepted by the majority is the one described by Dohan involving a single-low speed centrifugation with no activators or anticoagulants added. Taking into account variables such as fibrin network, the presence and the absence of leucocytes we have four different products: PRP, L-PRP (LP-PRP leukocyte-poor PRP), pure-platelet rich fibrin (P-PRF) and leucocyte and platelet rich fibrin (L-PRF). [10]

\section{Known facts and quality of evidence}

Despite the role of leucocytes in invalidating the bacterial growth, the fact that the myeloper- oxidate concentration was similar in products with and without leucocytes indicates the antibacterial role of platelets. [11] Nevertheless, bacterial growth inhibition is determined by the quality of PRP. Variables that influence the quality and quantity of PRP are: the number of donor's platelets, the type of activating and clotting agent, the number of centrifugation steps, the force of gravity, the validation of method with positive results from clinical trials. [12]

PRP contains growth factors such as: connective tissue growth factors (CTGF), epidermal growth factor (EGF), fibroblast growth factor - acidic (aFGF/FGF-1), fibroblast growth factor (bFGF/FGF-2), granulocyte/macrophage colony-stimulating factor (GM-CDF/CSF), insulin-like growth factor (IGF), keratinocyte growth factor (FGF-7), platelet-derived growth factor (PDGF), transforming growth factor alpha (TGF- $\alpha$ ), transforming growth factor beta (TGF-ß), vascular endothelial growth factor (VEGF/VEP), interleukin-1ß (IL-1ß), interleukin-8 (IL-8) and tumor necrosis factor alpha (TNF $\alpha$ ) supporting neurovascularization, angiogenesis, promoting mitogenic functions thus contributing to tissue healing. [9,12]

The presence of growth factors in PRP was shown in an experimental study that aimed the effects of PRP on healing a fascia in rats with fecal induced peritonitis. The levels of TNF $\alpha$ and TGF- $\beta$ were higher in fecal peritonitis treated fascia with PRP versus the control group. [14]

A meta-analysis that included 7 randomized control studies (RCTs) involved 1689 patients that underwent cardiac surgery, showed lower sternal infection versus controlled group after applying topical platelet gel: 11 events versus 66. Looking for deep sternal wound infections incidence, the results were similar with 7 events in the experimental group versus 33 complications in the control group. [3]

The main strains involved in nosocomial infections are E. coli, S. aureus, Streptococcus 
faecalis, Klebsiella pneumoniae and Pseudomonas aeruginosa. The group of Mariani studied the P-PRP effect in patients with nosocomial infections and as well the microbicidial proteins released by the platelet concentrate. They discovered a correlation between $S$ aureus and $S$ faecalis with increased IL-8. Staphylococcus aureus growth was associated with EGF. RANTES was associated with Pseudomonas aeruginosa, S. aureus and Sfaecalis. [15]

Another in vitro study aimed for the benefits of PRP in MRSA related surgical wound infection. The aimed groups were the ones treated with PRP with or without Vancomycin. The inflammatory scores were lowered in groups that associated PRP with or without Vancomycin vs only sham or Vancomycin treatment. [16]

The evidence for the benefit of platelets concentrates is not overhelming, but important enough to be discussed in the NICE guidelines (National Institute for Health and Care Excellence). (NICE). They recently concluded that intraarticular PRP was safe and effective for intraarticular injections, although the long term clinical effectiveness is unknown. [17]

\section{Perspectives}

Platelets concentrates can be used in the future in the protocols of wound infections, if standardization is to be considered.

\section{References}

1. Singer M, Deutschman CS, Seymour CW, Shankar-Hari M, Annane D, Bauer M, et al. The Third International Consensus Definitions for Sepsis and Septic Shock (Sepsis-3). JAMA. 2016;315(8):801-10. DOI: 10.1001/jama.2016.0287

2 Walkey A, Lagu T, Lindenauer PK. Trends in Sepsis and Infection Sources in the United States - A population base Study Ann Am Thorac Soc. 2015;12(2):21620. DOI: 10.1513/AnnalsATS.201411-498BC

3. Karman BH, Jones SG, Datta S, Mclaughlin EK,
Hoschtitzky AJ. A meta-analysis of platelet gel for prevention of sternal wound infections following cardiac surgery, Blood Transfus. 2017;15:57-65.

4. Çetinkaya RA, Yenilmez E, Petrone P, Yilmaz S, Bektöre B, Şimsek B, et al. Platelet-rich plasma as an additional therapeutic option for infected wounds with multi-drug resistant bacteria: in vitro antibacterial activity study. Eur J Trauma Emerg Surg. 2018. DOI: 10.1007/s00068-018-0957-0

5. Hamzeh-Cognasse H, Damien P, Chabert A, Pozzetto B, Cognasse F, Garraud O. Platelets and infections complex interactions with bacteria, Front Immunol. 2015;6(82):1-18. DOI: 10.3389/fimmu.2015.00082

6. Assinger A. Platelets and infection. An emerging role of platelets in viral infection. Front Immunol. 2014 Dec 18;5:649 DOI: 10.3389/fimmu.2014.00649

7. Costache A, Avram SI, Cernucan A, Barbu D, Angelescu S, Mut Popescu D, et al. What about microparticles? Perspectives and practical aspects. Rev Romana Med Lab. 2013;21(1):9-15. DOI:10.2478/rrlm-2013-0013

8. Cox D, Kerrigan SW, Watson SP. Platelets and the innate immune system: mechanism of bacterial-induced platelet activation. J Thromb Haemost. 2011;9: 1097-107. DOI: 10.1111/j.1538-7836.2011.04264.X

9. Yeaman MR, Yount NY, Waring AJ, Gank KD, Kupferwasser D, Wiese R, et al. Modular determinants of antimicrobial activity in platelet factor-4 family kinocidins. Biochim Biophys Acta. 2007;1768:67-90.

10. Lana JF, Weglein A, Vicente E, Perez AGM, Rodriguez AA, Luzo ACM, et al. Platelet Rich Plasma and Its Growth Factors: The State of Art, Lecture Notes in Bioengineering, 2014. DOI: 10.1007/978-3-642-40117-6

11. Mariani E, Canella V, Berlingeri A, Bielli A, Cattini L, Landini MP, et al. Leucocytes presence does not increase microbicidal activity of Platelet-rich Plasma in vitro, BMC Microbiology. 2015;15:149. DOI: 10.1186/ s12866-015-0482-9

12. Magshoudi O, Ranjbar R, Mirjalili SH, Fasihi-Ramandi M. Inhibitory activities of platelet-rich plasma and platelet-poor plasma on the growth of pathogenic bacteria. Iran J Pathol. 2017;12(1):79-87.

13. Fernandez-Moure J, Van Eps J, Cabrera F, Barbosa Z, Medrano Del Rosal G, Weiner BK, et al. Platelet-rich plasma: a biomimetic approach to enhancement of surgical wound healing. J Surg Res. 2017 Jan;207:33-44. DOI: 10.1016/j.jss.2016.08.063

14. Girgin M, Binnetoglu K, Duman K, Hakan Kanat B, 
Cetinkaya Z, Ayten R, et al. Effects of platelet rich plasma on fascial healing in rats with fecal peritonitis. Acta Cir Bras. 2016 May;31(5):314-9. DOI: 10.1590/S0102865020160050000004

15. Mariani E, Filardo G, Canella V, Berlingeri A, Bielli A, Cattini L, et al. Platelet-rich plasma affects bacterial growth in vitro. Cytotherapy. 2014 Sep;16(9):1294304. DOI: 10.1016/j.jcyt.2014.06.003
16. Çetinkaya, R.A., Yilmaz S, Únlú A, Petrone P, Marini C, Karabulut E, et al. The efficacy of platelet-rich plasma gel in MRSA-related surgical wound infection treatment: an experimental study in an animal model. Eur J Trauma Emerg Surg. 2017 Sep 30. DOI: 10.1007/ s00068-017-0852-0

17. https:/www.nice.org.uk/guidance/indevelopment/gidipg10077/documents accessed 2018 Sept. 